Article

\title{
On The Third-Order Complex Differential Inequalities of $\xi$-Generalized-Hurwitz-Lerch Zeta Functions
}

\author{
Hiba Al-Janaby ${ }^{1}$, Firas Ghanim ${ }^{2} \mathbb{C}$ and Maslina Darus ${ }^{3, *}$ (D) \\ 1 Department of Mathematics, College of Science, University of Baghdad, Baghdad 10071, Iraq; \\ fawzihiba@yahoo.com \\ 2 Department of Mathematics, College of Science, University of Sharjah, Sharjah, UAE; fgahmed@sharjah.ac.ae \\ 3 Department of Mathematical Sciences, Faculty of Science and Technology, Universiti Kebangsaan Malaysia, \\ Bangi 43600, Selangor, Malaysia \\ * Correspondence: maslina@ukm.edu.my
}

Received: 31 March 2020; Accepted: 2 May 2020; Published: 23 May 2020

check for updates

\begin{abstract}
In the $\mathrm{z}$ - domain, differential subordination is a complex technique of geometric function theory based on the idea of differential inequality. It has formulas in terms of the first, second and third derivatives. In this study, we introduce some applications of the third-order differential subordination for a newly defined linear operator that includes $\xi$-Generalized-Hurwitz-Lerch Zeta functions (GHLZF). These outcomes are derived by investigating the appropriate classes of admissible functions.
\end{abstract}

Keywords: holomorphic function; univalent function; $p$-valent function; convolution product; $\xi$-Generalized Hurwitz-Lerch Zeta function; differential subordination; admissible functions

MSC: 30C45; 33C10; 30C 80

\section{Introduction and Terminology}

Complex Function Theory (CFT) is a mathematical branch dating back to the 18th century. It investigates the functions of complex numbers. This branch has attracted the concern of several researchers. Among the remarkable names are Euler, Gauss, Riemann, Cauchy and others. It has numerous implementations in diverse fields of mathematics and science. These functions have many interesting properties that are not owned by real-valued functions. For instance, infinitely differentiable functions, holomorphic functions, every holomorphic function in the open unit disk can be represented as a Taylor series, conformal functions (that is, they preserve angles when $f^{\prime}(z) \neq 0$ ), line integrals, and all types of handy formulas. The considerable area in CFT is the Geometric Function Theory (GFT). The study of GFT includes investigating the interaction between the analytical properties of the complex holomorphic function and the geometrical properties of the image domain. Riemann [1] in 1851 introduced the first major result in GFT named the Riemann Mapping Theorem. Later, in 1907, Koebe [2] was a prominent scientist who studied the univalent functions in the open unit disk. Thereafter, in 1912, Koebe [3] presented a modified version of the Riemann's mapping theorem by utilized univalent functions. The theory tends towards the principle of "univalent" and "holomorphic", Riemann's mapping theorem plays a significant role in the collection of both principles. This synthesis interprets the formula of a domain where the complex functions being defined, for details see [1,4].

On the other hand, differential inequality theory (inequalities including derivatives of functions) impacted the development of GFT due to it giving much information regarding the behavior of the holomorphic function. Further, there are many differential implications in which characterization of a 
holomorphic function is settled by a differential condition. For instance, the Noshiro-Warschawski theorem states that for a holomorphic function in the unit disk, $\Re\left(f^{\prime}(z)\right)>0$ implies that $f$ is univalent function in the unit disk. Most of the known differential implications dealt with real-value inequalities that involved the absolute value, the imaginary part, or the real part of a complex function [4].

The principle of subordination is central to the theory of differential subordination of complex-valued function which is the generalizing the formula of differential inequality of real-valued function. Its origins back to Lindelöf [4] in 1909, though Littlewood ([5,6]) and Rogosinski ([7,8]) posed the term and examined the basic outcomes regarding subordination. This principle, as an advantageous tool, displays its importance to unify the presentation of several geometric classes in addition to achieving sandwich-type outcomes.

The methods of differential subordination are employed to study upper bounds for holomorphic functions in the unit disk. This technique inspired numerous researchers to work in GFT. The implementations and extensions of differential subordination theory have been developed in this theme and diverse other fields, such as functions of several complex variables, integral operator theory, meromorphic function theory, harmonic functions theory, differential equations and partial differential equations. Many papers handled the first-order and second-order differential subordination methods,but few articles handled the third-order differential subordination method. In 1935, Goluzin [9] studied the first significant outcome that includes the first-order differential subordination. Afterwards, Suffridge [10] in 1970 and Robinson [11] in 1947 discussed further successive investigations into first-order differential subordination. Later, in 1981, Miller and Mocanu [12] provided a systematic study of the theory of differential subordination. In 1985 [13] and 1987 [14], they evolved and studied several interesting outcomes on this theory. Next, numerous important studies were presented by Miller and Mocanu ([15-17]). In 1992, Ponnusamy and Juneja [18] considered the third-order inequalities and subordination. After that, in 2000, Miller and Mocano in their monograph [19] provided a marvelous and extensive discussion on this theory with numerous implementations.

In 2011, Antonino and Miller [20] investigated and extended the second-order differential subordination to the third-order case. Several authors provided fruitful implementations in the same direction of study. For their contributions, Tang et al. [21] considered some third-order differential subordination outcomes for meromorphically $p$-valent functions associated with the certain linear operator. At the same time, Tang and Deniz [22] studied a similar problem for holomorphic functions, involving the generalized Bessel functions. In 2015, Farzana et al. [23] introduced several third-order differential subordination outcomes for holomorphic functions associated with the fractional derivative operator. Related to this period, Tang et al. [24] used third-order differential subordination methods of holomorphic functions associated with generalized Bessel functions to yield sandwich-type outcomes containing this operator. In the same year, Ibrahim et al. [25] established some third-order differential subordination outcomes for holomorphic functions associated with a fractional integral operator (Carlson-Shaffer operator type). Subsequently, the problems of the third-order differential subordination were studied by El-Ashwah and Hassan [26], El-Ashwah and Hassan [27], Attiya et al. ([28,29]), Srivastava et al. [30] and Gochhayat and Prajapati [31]. Many of the studies have not yet been investigated utilizing third-order differential subordination technique. In this investigation, we impose a new generalized Noor-type linear integral operator $\mathcal{M}_{p}^{\ell} \vartheta(z)$ on the class $\mathcal{A}_{p}$ of $p$-valent functions by utilizing $\xi$-Generalized Hurwitz-Lerch Zeta functions (GHLZF). Some outcomes concerning an application of the third-order differential subordination for multivalent functions including operator $\mathcal{M}_{p}^{\ell} \vartheta(z)$ are studied.

Denote by $\mathbb{D}=\{z \in \mathbb{C}:|z|<1\}$ the open unit disc in the complex plane $\mathbb{C}$, and $\mathcal{H}(\mathbb{D})$ the class of holomorphic functions in $\mathbb{D}$. For $\alpha \in \mathbb{C}, \jmath \in \mathbb{N}=\{1,2,3, \ldots\}$, let

$$
\mathcal{H}[\alpha, \jmath]=\left\{\vartheta \in \mathcal{H}(\mathbb{D}): \vartheta(z)=\alpha+\alpha_{\jmath} z^{\jmath}+\alpha_{\jmath+1} z^{\jmath+1}+\ldots\right\}
$$


and suppose that $\mathcal{H}_{0} \equiv \mathcal{H}[0,1]$ and $\mathcal{H}_{1} \equiv \mathcal{H}[1,1]$. Let $\mathcal{A}$ denote the class of all holomorphic functions $\vartheta$ in $\mathbb{D}$, normalized by the conditions $\vartheta(0)=\vartheta^{\prime}(0)-1=0$, and of the formula

$$
\vartheta(z)=z+\sum_{j=2}^{\infty} \alpha_{\jmath} z^{\jmath}, \quad(z \in \mathbb{D}) .
$$

The subclass of $\mathcal{A}$ involving holomorphic univalent function is denoted by $\mathcal{S}$, [1]. In [4] the concept of subordination between holomorphic functions given as: for two functions $\vartheta_{1}$ and $\vartheta_{2}$, holomorphic in $\mathbb{D}$, the function $\vartheta_{1}$ is said to be subordinate to $\vartheta_{2}$, or $\vartheta_{2}$ superordinate to $\vartheta_{1}$ in $\mathbb{D}$, written $\vartheta_{1} \prec \vartheta_{2}$, if there is a holomorphic function $\hbar$ in $\mathbb{D}$ with $\hbar(0)=0$ and $|\hbar(z)|<1$ for all $z \in \mathbb{D}$, such that $\vartheta_{1}(z)=\vartheta_{2}(\hbar(z))$. In particular, if the function $\vartheta_{2}$ is univalent in $\mathbb{D}$, then the following characterization for subordination is gained as:

$$
\vartheta_{1} \prec \vartheta_{2} \text { if and only if } \vartheta_{1}(0)=\vartheta_{2}(0) \text { and } \vartheta_{1}(\mathbb{D}) \subset \vartheta_{2}(\mathbb{D}) \text {. }
$$

The natural generalization of holomorphic univalent function is a $p$-valent (multivalent) function, that is, if for each $\omega$, the equation $\vartheta(z)=\omega$ has at most $p$ roots in a domain $\mathfrak{D} \subset \mathbb{C}$, and if there is $\omega_{0}$ such that the equation $\vartheta(z)=\omega_{0}$ has exactly $p$ roots in a Domain $\mathfrak{D}$. Let $\mathcal{A}_{p}(p \in \mathbb{N}=\{1,2,3, \ldots\})$ denote the class involves all $p$-valent functions in $\mathbb{D}$ of the form

$$
\vartheta(z)=z^{p}+\sum_{j=p+1}^{\infty} \alpha_{\jmath} z^{\jmath}, \quad(z \in \mathbb{D}) .
$$

If $\vartheta$ is the $p$-valent function with $p=1$, then $\vartheta$ is the holomorphic univalent function, [4].

As one of the most remarkable tools, namely Hadamard (convolution) product, utilizes to formulate assorted operators: differential, integral and convolution operators. The term "Hadamard product" is attributed to Hadamard in 1899 [1] and defined as: for two functions $\vartheta_{\ell} \in \mathcal{A}$ of the form $\vartheta_{\ell}(z)=z+\sum_{\jmath=2}^{\infty} \alpha_{\jmath, \ell} z^{\jmath}, \ell=1,2$, their convolution, $\vartheta_{1} * \vartheta_{2}$, is given by

$$
\left(\vartheta_{1} * \vartheta_{2}\right)(z)=z+\sum_{j=2}^{\infty} \alpha_{j, 1} \alpha_{\jmath, 2} z^{\jmath}, \quad(z \in \mathbb{D}) .
$$

More generally, the convolution product of two functions $\vartheta_{\ell} \in \mathcal{A}_{p}$ of the formula $\vartheta_{\ell}(z)=$ $z^{p}+\sum_{\jmath=p+1}^{\infty} \alpha_{\jmath, \ell} z^{\jmath}, \ell=1,2, p \in \mathbb{N}$, is the function $\vartheta_{1} * \vartheta_{2}$ given by

$$
\left(\vartheta_{1} * \vartheta_{2}\right)(z)=z^{p}+\sum_{\jmath=p+1}^{\infty} \alpha_{\jmath, 1} \alpha_{\jmath, 2} z^{\jmath}, \quad(z \in \mathbb{D}) .
$$

In 1915, Alexander [32] was the first to introduce a linear integral operator which drafted in terms of the convolution, namely "Alexander operator" as follows: let $\vartheta \in \mathcal{A}$ and $I_{A}: \mathcal{A} \rightarrow \mathcal{A}$ be defined as

$$
\begin{aligned}
I_{A} \vartheta(z) & =\int_{0}^{z} \frac{\vartheta(t)}{t} d t=-\log (1-z) * \vartheta(z) \\
& =z+\sum_{j=2}^{\infty} \frac{\alpha_{j}}{\jmath} z^{\jmath}
\end{aligned}
$$


Later on, in 1965, Libera [33] given another linear integral operator so-called "Libera operator" $I_{L}: \mathcal{A} \rightarrow \mathcal{A}$ by the formula

$$
\begin{aligned}
I_{L} \vartheta(z) & =\frac{2}{z} \int_{0}^{z} \vartheta(t) d t=\frac{2[z+\log (1-z)]}{z} * \vartheta(z) \\
& =z+\sum_{j=2}^{\infty} \frac{2}{\jmath+1} \alpha_{j} z^{\jmath} .
\end{aligned}
$$

In 1969, Bernardi [34] imposed a more general linear integral operator $I_{\varepsilon}: \mathcal{A} \rightarrow \mathcal{A}$, for $\vartheta \in \mathcal{A}$ and $\varepsilon>-1$, as

$$
\begin{aligned}
I_{\varepsilon} \vartheta(z) & =\frac{1+\varepsilon}{z^{\varepsilon}} \int_{0}^{z} \vartheta(t) t^{\varepsilon} d t=z+\sum_{j=2}^{\infty}\left(\frac{\varepsilon+1}{\varepsilon+\jmath}\right) z^{\jmath} * \vartheta(z) \\
& =z+\sum_{j=2}^{\infty}\left(\frac{\varepsilon+1}{\varepsilon+\jmath}\right) \alpha_{\jmath} z^{\jmath} .
\end{aligned}
$$

The operator $I_{\varepsilon}$ is called the generalized Bernardi-Libera-Livingston integral operator. For $\varepsilon=0$, the operator $I_{\varepsilon}$ reduces to the Alexander operator $I_{A}$ given by Equation (6) and for $\varepsilon=1$, it reduces to the Libera operator $I_{L}$ defined by Equation (7).

Utilizing the convolution technique, in 1975, Ruscheweyh [35] proposed a linear operator as: let $\vartheta \in \mathcal{A}, \wp>-1$ and $D^{\wp}: \mathcal{A} \rightarrow \mathcal{A}$ be defined by

$$
D^{\wp} \vartheta(z)=\frac{z}{(1-z)^{\wp+1}} * \vartheta(z)=z+\sum_{\jmath=2}^{\infty} \frac{\Gamma(\wp+\jmath)}{\Gamma(\jmath) \Gamma(\wp+1)} \alpha_{\jmath} z^{\jmath} .
$$

For $\wp=\omega \in \mathbb{N}_{0}=\mathbb{N} \cup\{0\}$, yields

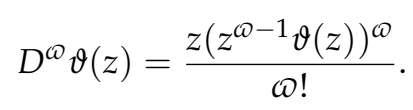

Further, $D^{0} \vartheta(z)=\vartheta(z)$ and $D^{\prime} \vartheta(z)=z \vartheta^{\prime}(z), z \in \mathbb{D}$. The operator $D^{\omega}$ is called the Ruscheweyh derivative of $\omega^{\text {th }}$ order of $\vartheta$.

Corresponding to the Ruscheweyh operator $D^{\omega}, \omega \in \mathbb{N}_{0}$ given by Equation (10), in 1999, Noor [36] considered the following linear operator: let $\vartheta \in \mathcal{A}, \omega \in \mathbb{N}_{0}$ and $I_{\boldsymbol{\omega}}: \mathcal{A} \rightarrow \mathcal{A}$ be defined as

$$
\begin{aligned}
I_{\mathscr{\omega}} \vartheta(z)=\vartheta_{\omega}^{(-1)}(z) * \vartheta(z) & =\left[\frac{z}{(1-z)^{\mathscr{\omega}+1}}\right]^{-1} * \vartheta(z) \\
& =z+\sum_{\jmath=2}^{\infty} \frac{\Gamma(\jmath+1) \Gamma(\omega+1)}{\Gamma(\omega+\jmath)} \alpha_{\jmath} z^{\jmath}
\end{aligned}
$$

such that

$$
\vartheta_{\mathcal{\omega}}(z) * \vartheta_{\omega}^{(-1)}(z)=\frac{z}{(1-z)^{2}} .
$$

Evidently, $I_{0} \vartheta(z)=z \vartheta^{\prime}(z), I_{1} \vartheta(z)=\vartheta(z), z \in \mathbb{D}$. This reverse relationship between the operators $I_{\omega}$ and $D^{\omega}$ gives a a cause for naming the Noor operator an integral operator. The operator $I_{\omega}$ is called as the Noor integral operator of $\omega^{\text {th }}$ order of $\vartheta$. 
Analogous to $D^{\wp}, \wp>-1$ written by Equation (9), in 2002, Choi, Saigo and Srivastava [37] defined the linear operator $I_{\wp, \mathfrak{F}}: \mathcal{A} \rightarrow \mathcal{A}$, for $\vartheta \in \mathcal{A}, \wp>-1$ and $\mathfrak{F}>0$ by

$$
\begin{aligned}
I_{\wp, \mathfrak{F}} \vartheta(z)=\vartheta_{\wp}^{-1}(z) * \vartheta(z) & =\left[\frac{z}{(1-z)^{\wp+1}}\right]^{-1} * \vartheta(z) \\
& =z+\sum_{\jmath=2}^{\infty} \frac{\Gamma(\mathfrak{F}+\jmath-1) \Gamma(\wp+1)}{\Gamma(\mathfrak{F}) \Gamma(\wp+\jmath)} \alpha_{\jmath} z^{\jmath},
\end{aligned}
$$

such that

$$
\vartheta_{\wp}(z) * \vartheta_{\wp}^{-1}(z)=\frac{z}{(1-z) \mathfrak{F}} .
$$

The operator $I_{\wp, \mathfrak{F}}$ is called the Choi-Saigo-Srivastava operator. For $\wp=\mathfrak{\omega}$ and $\mathfrak{F}=2$ reduces to the Noor integral operator $I_{\omega}$ of Equation (11).

In 2002, Liu and Noor [38] provided a linear operator as: for $\vartheta \in \mathcal{A}_{p}, \wp>-p$ and $I_{\wp+p}: \mathcal{A}_{p} \rightarrow \mathcal{A}_{p}$ defined by

$$
\begin{aligned}
I_{\wp+p} \vartheta(z)=\vartheta_{\wp+p}^{(-1)}(z) * \vartheta(z) & =\left[\frac{z^{p}}{(1-z)^{\wp+p}}\right]^{-1} * \vartheta(z) \\
& =z^{p}+\sum_{\jmath=p+1}^{\infty} \frac{\Gamma(1+\jmath) \Gamma(\wp+p)}{\Gamma(1+p) \Gamma(\wp+\jmath)} \alpha_{\jmath} z^{\jmath},
\end{aligned}
$$

such that

$$
\varphi_{\wp+p}(z) * \varphi_{\wp+p}^{(-1)}(z)=\frac{z^{p}}{(1-z)^{p+1}} .
$$

Obviously, $I_{0+p} \vartheta(z)=z \vartheta^{\prime}(z) / p$ and $I_{1+p} \vartheta(z)=\vartheta(z)$. The operator $I_{\wp+p}$ is an extended Noor integral operator $I_{\varpi}$ of Equation (11). In addition, the operator $I_{\wp+p}$ is closely related to the Choi-Saigo-Srivastava operator $I_{\wp, \mathfrak{F}}$ of Equation (12).

The Theory of Hypergeometric Functions (HFT) has been incorporated in GFT. Employing hypergeometric functions in the proof of the famed problem "Bieberbach conjecture" by de Branges in 1984 [39] has given complex analysts a renewed attention to study the role of special functions. In this regard a lot of implementations and generalizations are found. The study of this theory gained an independent status. The Gauss Hypergeometric Function (GHF), denoted by $\mathcal{F}(\mu, v ; \tau ; \omega)$, was first introduced by Gauss in 1812 [39]. It is given as follows: for $\mu, v$ and $\tau$ be complex numbers with $\tau$ other than $0,-1,-2, \ldots$, and

$$
\mathcal{F}(\mu, v ; \tau ; z)=\sum_{j=0}^{\infty} \frac{(\mu)_{\jmath}(v)_{\jmath}}{(\tau)_{\jmath}(1)_{j}} z^{\jmath}=1+\frac{\mu \nu}{\tau} z+\frac{\mu(\mu+1) v(v+1)}{\tau(\tau+1)} \frac{z^{2}}{2 !}+\ldots
$$

where $(\varrho)_{j}$ is the Pochhammer symbol given by

$$
(\varrho)_{\jmath}:=\frac{\Gamma(\varrho+\jmath)}{\Gamma(\varrho)}=\left\{\begin{array}{l}
1, \\
\varrho(\varrho+1)(\varrho+2) \ldots(\varrho+\jmath-1), \quad(\jmath \in \mathbb{N}=\{1,2,3, \ldots\}) .
\end{array}\right.
$$


Another important special function related to GHF is the incomplete beta function $\varphi_{p}(\mu, \tau ; z)$ defined (for $\mu \in \mathbb{R}, \tau \in \mathbb{R} \backslash \mathbb{Z}_{0}^{-}, \mathbb{Z}_{0}^{-}=\{\ldots,-2,-1,0\}$ ) by

$$
\varphi_{p}(\mu, \tau ; z)=z \mathcal{F}(\mu, 1 ; \tau ; z)=\sum_{j=0}^{\infty} \frac{(\mu)_{j}}{(\tau)_{j}} z^{j+p}
$$

Other generalized Noor-type linear integral operators between classes of holomorphic functions associated with hypergeometric functions and its generalizations have been posed by authors. For instance, Al-Janaby et al. ([40,41]).

Recently, the theory of Hurwitz-Lerch Zeta functions has a fruitful role in the study operators. This theory is developed with numerous implementations and generalizations by various researchers. One may refer to Al-Janaby et al. [42,43], Ghanim [44], Ghanim and Darus [45], Ghanim and Al-Janaby [46], Răducanu and Srivastava [47], Srivastava and Attiya [48], Srivastava et al. [49,50], Xing and Jose [51], Choi and Srivastava [52], Milovanovic and Rassias [53] and Rassias and Yang [54-57].

In terms of the Hurwitz-Lerch Zeta function $\Phi(z, \gamma, \eta)$ defined by (see, for example, [58-60])

$$
\begin{gathered}
\Phi(z, \gamma, \eta):=\sum_{j=0}^{\infty} \frac{z^{\jmath}}{(j+\eta)^{\gamma}} \\
\left(\eta \in \mathbb{C} \backslash \mathbb{Z}_{0}^{-}, \gamma \in \mathbb{C} \text { when }|z|<1,1<\Re(\gamma) \text { when }|z|=1\right) .
\end{gathered}
$$

The following new family of the (GHLZF) was considered systematically by Srivastava [61]:

$$
\begin{gathered}
\Phi_{\mu_{1}, \cdots, \mu_{r}, \nu_{1}, \cdots, \nu_{s}}^{\left(\rho_{1}, \cdots, \rho_{r}, \sigma_{1}, \cdots, \sigma_{s}\right)}(z, \gamma, \eta ; \zeta, \xi) \\
=\frac{1}{\xi \Gamma(\gamma)} \sum_{j=0}^{\infty} \frac{\prod_{i=1}^{r}\left(\mu_{i}\right)_{j \rho_{i}}}{(\eta+\jmath)^{\gamma} \cdot \prod_{i=1}^{s}\left(v_{i}\right)_{j \sigma_{i}}} H_{0,2}^{2,0}\left[(\eta+\jmath) \zeta^{\frac{1}{\xi}} \mid \overline{(\gamma, 1),\left(0, \frac{1}{\zeta}\right)}\right] \frac{z^{\jmath}}{\jmath !} \\
(0<\min \{\Re(\eta), \Re(\gamma)\}, 0<\Re(\zeta) ; 0<\xi),
\end{gathered}
$$

where

$$
\begin{gathered}
\left(\mu_{i} \in \mathbb{C}(i=1, \cdots, p), v_{i} \in \mathbb{C} \backslash \mathbb{Z}_{0}^{-}(i=1, \cdots, s), 0<\rho_{i}(i=1, \cdots, r),\right. \\
\left.0<\sigma_{i}(i=1, \cdots, q), \text { and } 0 \leqq 1+\sum_{i=1}^{s} \sigma_{i}-\sum_{i=1}^{r} \rho_{i}\right)
\end{gathered}
$$

and the equality in the convergence condition holds true for suitably bounded values of $|z|$ given by

$$
|z|<\nabla:=\left(\prod_{i=1}^{r} \rho_{i}^{-\rho_{i}}\right)\left(\prod_{i=1}^{s} \sigma_{i}^{\sigma_{i}}\right) .
$$

Definition 1. The H-function involved in the right-hand side of Equation (17) is the well-known Fox's H-function ([62], Definition 1.1) (see also [30,63]) defined by

$$
\begin{aligned}
H_{\mathfrak{p}, \mathfrak{q}}^{m, n}(z) & =H_{\mathfrak{p}, \mathfrak{q}}^{m, n}\left[z \mid \begin{array}{c}
\left(a_{1}, A_{1}\right), \cdots,\left(a_{\mathfrak{p}}, A_{\mathfrak{p}}\right) \\
\left(b_{1}, B_{1}\right), \cdots,\left(b_{\mathfrak{q}}, B_{\mathfrak{q}}\right)
\end{array}\right] \\
& =\frac{1}{2 \pi \mathrm{i}} \int_{\mathcal{L}} \Xi(\gamma) z^{-\gamma} \mathrm{d} \gamma \quad(z \in \mathbb{C} \backslash\{0\},|\arg (z)|<\pi),
\end{aligned}
$$

an empty product is interpreted as $1, m, n, \mathfrak{p}$ and $\mathfrak{q}$ are integers such that

$$
1 \leqq m \leqq \mathfrak{q} \quad \text { and } \quad 0 \leqq n \leqq \mathfrak{p},
$$




$$
\begin{aligned}
& 0<A_{i} \quad(i=1, \cdots, \mathfrak{p}) \quad \text { and } \quad 0<B_{i} \quad(i=1, \cdots, \mathfrak{q}) \text {, } \\
& a_{i} \in \mathbb{C} \quad(i=1, \cdots, \mathfrak{p}) \quad \text { and } \quad b_{i} \in \mathbb{C} \quad(i=1, \cdots, \mathfrak{q})
\end{aligned}
$$

and $\mathcal{L}$ is a suitable Mellin-Barnes type contour separating the poles of the gamma functions

$$
\left\{\Gamma\left(b_{i}+B_{i} \gamma\right)\right\}_{i=1}^{m}
$$

from the poles of the gamma functions

$$
\left\{\Gamma\left(1-a_{i}+A_{i} \gamma\right)\right\}_{i=1}^{n}
$$

It is worthy of mention here that, by using the fact that ([61], p. 1496, Remark 7)

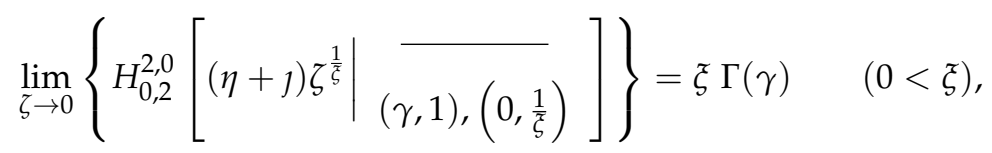

Equation (17) reduces to the following form:

$$
\begin{aligned}
\Phi_{\mu_{1}, \cdots, \mu_{r} ; \nu_{1}, \cdots, v_{s}}^{\left(\rho_{1}, \cdots, \rho_{s}, \sigma_{1}, \cdots, \gamma\right.}(z, \gamma ; 0, \xi) & :=\Phi_{\mu_{1}, \cdots, \mu_{r} ; \nu_{1}, \cdots, v_{s}}^{\left(\rho_{1}, \cdots, \rho_{s}, \sigma_{1}, \cdots, \gamma\right.}(z, \eta) \\
& =\sum_{j=0}^{\infty} \frac{\prod_{i=1}^{r}\left(\mu_{i}\right)_{j \rho_{i}}}{(\eta+\jmath)^{\gamma} \cdot \prod_{i=1}^{s}\left(v_{i}\right)_{j \sigma_{i}}} \frac{z^{\prime}}{\jmath !} .
\end{aligned}
$$

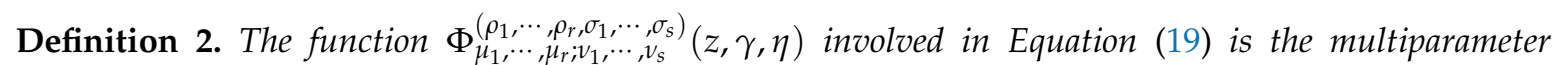
extension and generalization of the Hurwitz-Lerch Zeta function $\Phi(z, \gamma, \eta)$ introduced by Srivastava et al. ([64], p. 503, Equation (6.2)) defined by

$$
\begin{gathered}
\Phi_{\mu_{1}, \cdots, \mu_{r} ; \nu_{1}, \cdots, \nu_{s}}^{\left(\rho_{1}, \cdots, \rho_{s}, \sigma_{1}, \ldots, \gamma\right)}\left(z, \gamma, \sum_{j=0}^{\infty} \frac{\prod_{i=1}^{r}\left(\mu_{i}\right)_{\jmath \rho_{i}}}{(\eta+\jmath)^{\gamma} \cdot \prod_{i=1}^{s}\left(v_{i}\right)_{j \sigma_{i}}} \frac{z^{\jmath}}{j !}\right. \\
\left(r, s \in \mathbb{N}_{0} ; \mu_{j} \in \mathbb{C}(j=1, \cdots, r) ; \eta, v_{j} \in \mathbb{C} \backslash \mathbb{Z}_{0}^{-}(j=1, \cdots, s) ;\right. \\
\rho_{i}, \sigma_{i} \in \mathbb{R}^{+}(i=1, \cdots, r ; i=1, \cdots, s) ; \\
\quad-1<\Delta \text { when } \gamma, z \in \mathbb{C} ; \\
\Delta=-1 \text { and } \gamma \in \mathbb{C} \text { when }|z|<\nabla^{*} ; \\
\left.\Delta=-1 \text { and } \frac{1}{2}<\Re(\Xi) \text { when }|z|=\nabla^{*}\right)
\end{gathered}
$$

with

$$
\begin{gathered}
\nabla^{*}:=\left(\prod_{i=1}^{r} \rho_{i}^{-\rho_{j}}\right) \cdot\left(\prod_{i=1}^{s} \sigma_{i}^{\sigma_{i}}\right) \\
\Delta:=\sum_{i=1}^{s} \sigma_{i}-\sum_{i=1}^{r} \rho_{i} \text { and } \Xi:=t+\sum_{i=1}^{s} v_{i}-\sum_{i=1}^{r} \mu_{i}+\frac{r-s}{2} .
\end{gathered}
$$


In GFT, the third-order differential subordination methodology for holomorphic functions is indicated by Antonion and Miller [20], which is required in this investigation.

Definition 3 ([20], Definition 2, p. 441). Let $\mathcal{J}$ denote the set of holomorphic functions $\omega$ that are univalent on the set $\overline{\mathbb{D}} \backslash \mathcal{G}(\omega)$, where

$$
\mathcal{G}(\omega)=\left\{\chi \in \partial \mathbb{D}: \lim _{z \rightarrow \chi} \omega(z)=\infty\right\}
$$

is such that

$$
\min \left|\omega^{\prime}(\chi)\right|=\delta>0
$$

for $\chi \in \partial \mathbb{D} \backslash \mathcal{G}(\omega)$. Further, let $\mathcal{J}(\alpha)=\{\omega(z) \in \mathcal{J}: \omega(0)=\alpha\}, \mathcal{J}(0)=\mathcal{J}_{0}$ and $\mathcal{J}(1)=\mathcal{J}_{1}$.

Definition 4 ([20], Definition 1, p. 440). Let $\Gamma: \mathbb{C}^{4} \times \mathbb{D} \longrightarrow \mathbb{C}$ and the function $\pi(z)$ be univalent in $\mathbb{D}$. If the function $v(z)$ is holomorphic in $\mathbb{D}$ and satisfies the following third-order differential subordination:

$$
\Gamma\left(v(z), z v^{\prime}(z), z^{2} v^{\prime \prime}(z), z^{3} v^{\prime \prime \prime}(z) ; z\right) \prec \pi(z),
$$

then $v(z)$ is called a solution of the differential subordination. A univalent function $\omega(z)$ is called a dominant of the solutions of the differential subordination, or, more simply, a dominant if $v(z) \prec \omega(z)$ for all $v(z)$ achieving Equation (21). A dominant $\widetilde{\omega}(z)$ that achieves $\widetilde{\omega}(z) \prec \omega(z)$ for all dominants $\omega(z)$ of Equation (21) is said to be the best dominant.

The class of admissible functions related to differential subordination is presented next.

Definition 5 ([20], Definition 2, p. 449). Let $\Lambda$ be a set in $\mathbb{C}, \omega \in \mathcal{J}$ and $\mathcal{1} \in \mathbb{N} \backslash\{1\}$. The class of admissible functions denoted by $\Omega_{j}[\Lambda, \omega]$ consists of those functions $\Gamma: \mathbb{C}^{4} \times \mathbb{D} \longrightarrow \mathbb{C}$ that achieves the following admissibility condition:

$$
\Gamma(f, q, x, y ; z) \notin \Lambda
$$

whenever

$$
f=\omega(\chi), q=\kappa \zeta \omega^{\prime}(\chi), \Re\left(\frac{x}{q}+1\right) \geq \kappa \Re\left(\frac{\zeta \omega^{\prime \prime}(\chi)}{\omega^{\prime}(\chi)}+1\right)
$$

and

$$
\Re\left(\frac{y}{q}\right) \geq \kappa^{2} \Re\left(\frac{\chi^{2} \omega^{\prime \prime \prime}(\chi)}{\omega^{\prime}(\chi)}\right)
$$

where $z \in \mathbb{D}, \chi \in \partial \mathbb{D} \backslash \mathcal{G}(\omega)$, and $\kappa \geq \jmath$.

The following theorem is a key outcome in third-order differential subordination.

Theorem 1 ([20], Definition 2, p. 449). Let $v \in \mathcal{H}[\alpha, \jmath]$ with $\jmath \geq 2$, and let $\omega \in \mathcal{J}(\alpha)$ and achieve the following conditions:

$$
\Re\left(\frac{\chi \omega^{\prime \prime}(\chi)}{\omega^{\prime}(\chi)}\right) \geq 0 \text {, and }\left|\frac{z v^{\prime}(z)}{\omega^{\prime}(\chi)}\right| \leq \kappa,
$$


where $z \in \mathbb{D}, \chi \in \partial \mathbb{D} \backslash \mathcal{G}(\omega)$ and $\kappa \geq$ J. If $\Lambda$ is a set in $\mathbb{C}, \Gamma \in \Omega_{\jmath}[\Lambda, \omega]$ and

$$
\Gamma\left(v(z), z v^{\prime}(z), z^{2} v^{\prime \prime}(z), z^{3} v^{\prime \prime \prime}(z) ; z\right) \in \Lambda,
$$

then

$$
v(z) \prec \omega(z) \quad(z \in \mathbb{D}) .
$$

\section{Imposed Linear Integral Operator $\mathcal{M}_{p}^{\ell} \vartheta(z)$}

This section considers a new generalized Noor-type linear integral operator $\mathcal{M}_{p}^{\ell} \vartheta(z)$ for $p$-valent functions associated with the GHLZF in $\mathbb{D}$ defined in Equation (17). Setting $\rho_{1}=\cdots, \rho_{r}=\sigma_{1}=\cdots=$ $\sigma_{s}=1$, and $\mu_{i} \in \mathbb{C} \backslash \mathbb{Z}_{0}^{-}(i=1, \cdots, r)$ as follows:

$$
\begin{aligned}
& \Phi_{\mu_{1}, \cdots, \mu_{r}, v_{1}, \cdots, v_{s}}^{(1, \cdots, 1,1, \cdots, 1)}(z, \gamma, \eta ; \zeta, \xi) \\
& =\frac{1}{\xi \Gamma(\gamma)} \sum_{j=0}^{\infty} \frac{\prod_{i=1}^{r}\left(\mu_{i}\right)_{j}}{(\eta+\jmath)^{\gamma} \cdot \prod_{i=1}^{s}\left(v_{i}\right)_{j}} H_{0,2}^{2,0}\left[(\eta+\jmath) \zeta^{\frac{1}{\xi}} \mid \overline{(\gamma, 1),\left(0, \frac{1}{\zeta}\right)}\right] \frac{z^{\jmath}}{\jmath !} \\
& =\frac{1}{\xi \Gamma(\gamma)} \sum_{j=p}^{\infty} \frac{\prod_{i=1}^{r}\left(\mu_{i}\right)_{j}-p}{(\eta+(\jmath-p))^{\gamma} \cdot \prod_{i=1}^{s}\left(v_{i}\right)_{j-p}} H_{0,2}^{2,0}\left[(\eta+(\jmath-p)) \zeta^{\frac{1}{\xi}} \mid \overline{(\gamma, 1),\left(0, \frac{1}{\zeta}\right)}\right] \frac{z^{\jmath-p}}{(\jmath-p) !} .
\end{aligned}
$$

Thus, from Equation (22), we derive a new function as:

$$
\begin{aligned}
& \Psi_{\mu_{1}, \ldots, \mu_{r}}^{v_{1}, \ldots, \nu_{s}}(\gamma, \eta ; \zeta, \xi, \wp):=\xi \Gamma(\gamma) \mathrm{Y}\left[z^{p} \Phi_{\mu_{1}, \cdots, \mu_{r} ; \nu_{1}, \cdots, \nu_{s}}^{(1, \cdots, 1,1, \gamma, \eta ; \zeta, \xi)}(z)\right] \\
& =z^{p}+\sum_{\jmath=p+1}^{\infty} \frac{\mathrm{Y} \prod_{i=1}^{r}\left(\mu_{i}\right)_{\jmath}-p}{(\eta+(\jmath-p))^{\gamma} \cdot \prod_{i=1}^{s}\left(v_{i}\right)_{\jmath-p}} H_{0,2}^{2,0}\left[(\eta+(\jmath-p)) \zeta^{\frac{1}{\xi}} \mid \overline{(\gamma, 1),\left(0, \frac{1}{\xi}\right)}\right] \frac{z^{\prime}}{(\jmath-p) !}
\end{aligned}
$$

where $\mathrm{Y}$ is defined as:

$$
Y=\frac{\eta^{\gamma}}{H_{0,2}^{2,0}\left[\eta \zeta^{\frac{1}{\xi}} \mid \overline{(\gamma, 1),\left(0, \frac{1}{\xi}\right)}\right]}
$$


By employing the principle of convolution product of $\ell^{\text {th }}$ order of GHLZF, we yield

$$
\begin{aligned}
\mathcal{N}_{p, \mu_{1}, \ldots, \mu_{r}}^{\ell, \nu_{1}, \ldots, \nu_{s}}(\gamma, \eta ; \zeta, \xi, \wp): & =\underbrace{\Psi_{\mu_{1}, \ldots, \mu_{r}}^{v_{1}, \ldots, \nu_{s}}(\gamma, \eta ; \zeta, \xi, \wp) * \ldots * \Psi_{\mu_{1}, \ldots, \mu_{r}}^{v_{1}, \ldots, \nu_{s}}(\gamma, \eta ; \zeta, \xi, \wp)}_{\ell-\text { times }} \\
& =z^{p}+\sum_{j=p+1}^{\infty}\left[\frac{\mathrm{Y} \prod_{i=1}^{r}\left(\mu_{i}\right)_{j-p} H_{0,2}^{2,0}\left[(\eta+(\jmath-p)) \zeta^{\frac{1}{\xi}} \mid \overline{(\gamma, 1),\left(0, \frac{1}{\zeta}\right)}\right]}{(\eta+(j-p))^{\gamma} \prod_{i=1}^{s}\left(v_{i}\right)_{j-p}(j-p) !}\right]^{\ell} .
\end{aligned}
$$

Next, we present a new function $\left(\mathcal{N}_{p, \mu_{1}, \ldots, \mu_{r}}^{\ell, \nu_{1}, \ldots, v_{s}}(\gamma, \eta ; \zeta, \xi, \wp)\right)^{-1}$ given by

$$
\begin{aligned}
& \left(\mathcal{N}_{p, \mu_{1}, \ldots, \mu_{r}}^{\ell, \nu_{1}, \ldots, v_{s}}(\gamma, \eta ; \zeta, \xi, \wp)\right)^{-1} \\
& =z^{p}+\sum_{j=p+1}^{\infty}\left[\frac{(\eta+(\jmath-p))^{\gamma} \prod_{i=1}^{s}\left(v_{i}\right)_{j-p}(\jmath-p) !}{\mathrm{Y} \prod_{i=1}^{r}\left(\mu_{i}\right)_{j-p} H_{0,2}^{2,0}\left[(\eta+(\jmath-p)) \zeta^{\frac{1}{\xi}} \mid \overline{(\gamma, 1),\left(0, \frac{1}{\xi}\right)}\right.}\right]^{\ell}\left[\frac{(\wp+p)_{j-p}}{(\jmath-p) !}\right] z^{\prime},
\end{aligned}
$$

such that,

$$
\begin{aligned}
\left(\mathcal{N}_{p, \mu_{1}, \ldots, \mu_{r}}^{\ell, \nu_{1}, \ldots, v_{s}}(\gamma, \eta ; \zeta, \xi, \wp)\right) *\left(\mathcal{N}_{p, \mu_{1}, \ldots, \mu_{r}}^{\ell, v_{1}, \ldots, \nu_{s}}(\gamma, \eta ; \zeta, \xi, \wp)\right)^{-1} & =\frac{z^{p}}{(1-z)^{\wp+p}} \\
& =z^{p}+\sum_{j=p+1}^{\infty} \frac{(\wp+p)_{j-p}}{(\jmath-p) !} z^{\jmath}, \quad(\wp>-1) .
\end{aligned}
$$

Therefore, from Equation (26), we consider the following linear operator: $\mathcal{M}_{p, \mu_{1}, \ldots, \mu_{r}}^{\ell, \nu_{1}, \ldots, \nu_{s}}(\gamma, \eta ; \zeta, \xi, \wp)$ : $\mathcal{A}_{p} \longrightarrow \mathcal{A}_{p}$, which is defined by

$$
\begin{aligned}
& \mathcal{M}_{p, \mu_{1}, \ldots, \mu_{r}}^{\ell, v_{1}, \ldots, v_{s}}(\gamma, \eta ; \zeta, \xi, \wp) \vartheta(z)=\left(\mathcal{N}_{p, \mu_{1}, \ldots, \mu_{r}}^{\ell, v_{1}, \ldots, \nu_{s}}(\gamma, \eta ; \zeta, \xi, \wp)\right)^{-1} * \vartheta(z) \\
& =z^{p}+\sum_{\jmath=p+1}^{\infty}\left[\frac{(\eta+(\jmath-p))^{\gamma} \prod_{i=1}^{s}\left(v_{i}\right)_{\jmath-p}(\jmath-p) !}{\mathrm{Y} \prod_{i=1}^{r}\left(\mu_{i}\right)_{\jmath-p} H_{0,2}^{2,0}\left[(\eta+(\jmath-p)) \zeta^{\frac{1}{\xi}} \mid \overline{(\gamma, 1),\left(0, \frac{1}{\zeta}\right)}\right.}\right]^{\ell}\left[\frac{(\wp+p)_{\jmath-p}}{(\jmath-p) !}\right] \alpha_{\jmath} z^{\jmath} .
\end{aligned}
$$

Remark 1. For suitably specializing the parameters of $\ell, p, \zeta, \xi, \gamma, \eta, s, r, v_{i}$ and $\mu_{i}$, the operator $\mathcal{M}_{p}^{\ell} \vartheta(z)$ defined in Equation (27) can be reduced to various operators previously mentioned. Thus, we have the following special cases:

1. For $\ell=p=\gamma=\eta=1, \zeta=0, s=1, v_{1}=1, r=2, \mu_{1}=2$ and $\mu_{2}=1$ in Equation (27), we yield the Ruscheweyh operator given in Equation (9).

2. For $\ell=p=\gamma=\eta=1, \zeta=0, s=2, \nu_{1}=v_{2}=1, r=3, \mu_{1}=2$ and $\mu_{2}=\mu_{3}=\wp+1$, the operator Equation (27) reduce to the Noor operator defined by Equation (11).

3. For $\ell=\gamma=1, \zeta=0, s=2, v_{1}=\eta, v_{2}=1, r=3, \mu_{1}=\eta+1 \mu_{2}=\mu_{2}=\wp+p$, the operator Equation (27), we have the extended Noor operator given by Equation (13).

4. For $\ell=1, \zeta=0, s=2, \gamma=1, \nu_{1}=\eta, v_{2}=\tau, r=3, \mu_{1}=\eta+1, \mu_{2}=1$, and $\mu_{3}=\mu$, the operator Equation (27) provides the Noor-type integral operator defined by [65]. 
5. For $\ell=p=\gamma=\eta=1, \zeta=0, s=2, v_{1}=1, v_{2}=\tau, r=3, \mu_{1}=2, \mu_{2}=\mu$, and $\mu_{3}=v$, the operator Equation (27) provides the Noor integral operator given in [66].

6. For $\ell=p=\gamma=\eta=1, \zeta=0, s=1, v_{1}=\tau, r=3, \mu_{1}=2, \mu_{2}=\mu$, and $\mu_{3}=v$, the operator Equation (27) reduce to the generalized Noor-type linear integral operator defined in [67].

7. For $\ell=p=\gamma=\eta=1, \zeta=0 s=2, v_{1}=v_{2}=1, r=3, \mu_{1}=\mu_{2}=2$ and $\mu_{3}=\wp+1$, the operator Equation (27) reduce to Alexander operator given in Equation (6).

8. For $\ell=p=\gamma=\eta=1, \zeta=0 s=1, v_{1}=1, r=2, \mu_{1}=2$ and $\mu_{2}=\wp+1$, the operator Equation (27) is reduced to $\vartheta(z)$ given by Equation (2).

For convenience, Equation (27) is written as

$$
\mathcal{M}_{p}^{\ell} \vartheta(z) \equiv \mathcal{M}_{p, \mu_{1}, \ldots, \mu_{r}}^{\ell, v_{1}, \ldots, v_{r}}(\gamma, \eta ; \zeta, \xi, \wp) \vartheta(z) .
$$

This operator achieves the differential recurrence relation

$$
\frac{A z}{p}\left[\mathcal{M}_{p}^{\ell} \vartheta(z)\right]^{\prime}=\mathcal{M}_{p}^{\ell+1} \vartheta(z)-(1-A) \mathcal{M}_{p}^{\ell} \vartheta(z)
$$

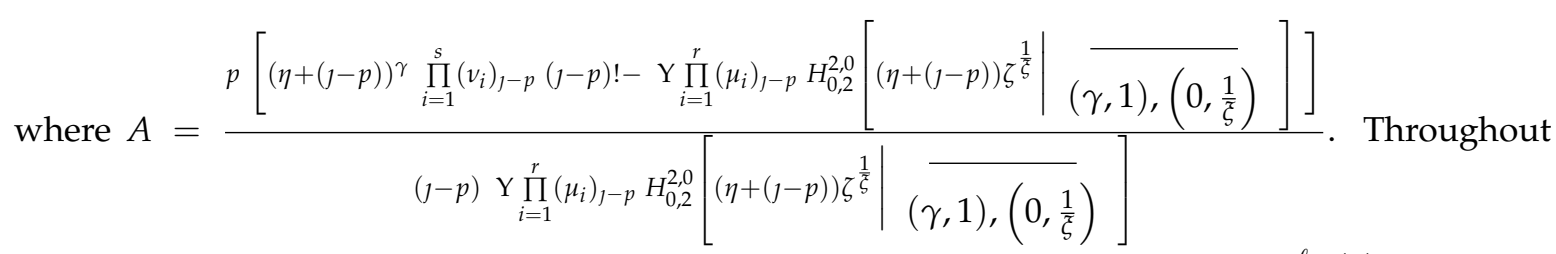
this paper, the generalized Noor-type linear integral operator will be denoted by $\mathcal{M}_{p}^{\ell} \vartheta(z)$.

\section{Differential Subordination with $\mathcal{M}_{p}^{\ell} \vartheta(z)$}

This section introduces certain appropriate class of admissible functions and studies some third-order differential subordination outcomes for the operator $\mathcal{M}_{p}^{\ell} \vartheta(z)$ defined by Equation (27).

Definition 6. Let $\mathfrak{A}$ be a set in $\mathbb{C}, \omega \in \mathcal{J}_{0}$ and $] \in \mathbb{N} \backslash\{1\}$. The class of admissible functions $\Sigma_{\mathcal{M}}[\mathfrak{A}, \omega]$ consists of those functions $¥: \mathbb{C}^{4} \times \mathbb{D} \longrightarrow \mathbb{C}$ that satisfy the following admissibility condition:

$$
¥\left(u_{1}, u_{2}, u_{3}, u_{4} ; z\right) \notin \mathfrak{A}
$$

whenever

$$
\begin{gathered}
u_{1}=\omega(\chi), \quad u_{2}=\frac{\kappa \chi \omega^{\prime}(\chi)+\frac{p(1-A)}{A} \omega(\chi)}{\frac{p}{A}}, \\
\Re\left(\frac{p\left[u_{3}-2(1-A) u_{2}+(1-A)^{2} u_{1}\right]}{A\left[u_{2}-(1-A) u_{1}\right]}\right) \geq \kappa \Re\left(\frac{\zeta \omega^{\prime \prime}(\chi)}{\omega^{\prime}(\chi)}+1\right),
\end{gathered}
$$

and

$\Re\left(\frac{p^{2}\left[u_{4}-(1-A)^{3} u_{1}\right]-p[3 A+3 p(1-A)]\left[u_{3}-2(1-A) u_{2}+(1-A)^{2} u_{1}\right]}{A^{2}\left[u_{2}-(1-A) u_{1}\right]}-\frac{3 p^{2}(1-A)^{2}}{A^{2}}+2\right)$ $\geq \kappa^{2} \Re\left(\frac{\chi^{2} \omega^{\prime \prime \prime}(\chi)}{\omega^{\prime}(\chi)}\right)$,

where $z \in \mathbb{D}, \chi \in \partial \mathbb{D} \backslash \mathcal{G}(\omega)$, and $\kappa \geq 1$. 
Theorem 2. Let $¥ \in \Sigma_{\mathcal{M}}[\mathfrak{A}, \omega]$. If $\vartheta \in \mathcal{A}_{p}$ and $\omega \in \mathcal{J}_{0}$ achieve the following conditions:

$$
\Re\left(\frac{\chi \omega^{\prime \prime}(\chi)}{\omega^{\prime}(\chi)}\right) \geq 0, \quad\left|\frac{\mathcal{M}_{p}^{\ell+1} \vartheta(z)-(1-A) \mathcal{M}_{p}^{\ell} \vartheta(z)}{\omega^{\prime}(\chi)}\right| \leq \frac{|A| \kappa}{p}
$$

and

$$
\left\{¥\left(\mathcal{M}_{p}^{\ell} \vartheta(z), \mathcal{M}_{p}^{\ell+1} \vartheta(z), \mathcal{M}_{p}^{\ell+2} \vartheta(z), \mathcal{M}_{p}^{\ell+3} \vartheta(z) ; z\right): z \in \mathbb{D}\right\} \subset \mathfrak{A},
$$

then

$$
\mathcal{M}_{p}^{\ell} \vartheta(z) \prec \omega(z), \quad(z \in \mathbb{D}) .
$$

Proof. Define the following holomorphic function $v(z)$ in $\mathbb{D}$ by

$$
v(z)=\mathcal{M}_{p}^{\ell} \vartheta(z) .
$$

From Equations (29) and (33), we have

$$
\mathcal{M}_{p}^{\ell+1} \vartheta(z)=\frac{z v^{\prime}(z)+\frac{p(1-A)}{A} v(z)}{\frac{p}{A}} .
$$

Further computations show that

$$
\mathcal{M}_{p}^{\ell+2} \vartheta(z)=\frac{z^{2} v^{\prime \prime}(z)+\left[1+\frac{2 p(1-A)}{A}\right] z v^{\prime}(z)+\frac{p^{2}(1-A)^{2}}{A^{2}} v(z)}{\frac{p^{2}}{A^{2}}},
$$

and

$\mathcal{M}_{p}^{\ell+3} \vartheta(z)=\frac{z^{3} v^{\prime \prime \prime}(z)+\left[3+\frac{3 p(1-A)}{A}\right] z^{2} v^{\prime \prime}(z)+\left[1+\frac{3 p(1-A)}{A}+\frac{3 p^{2}(1-A)^{2}}{A^{2}}\right] z v^{\prime}(z)+\frac{p^{3}(1-A)^{3}}{A^{3}} v(z)}{\frac{p^{3}}{A^{3}}}$.

Define the parameters $u_{1}, u_{2}, u_{3}$ and $u_{4}$ as:

$$
\begin{gathered}
u_{1}=f, \quad u_{2}=\frac{g+\frac{p(1-A)}{A} f}{\frac{p}{A}}, \\
u_{3}=\frac{h+\left[1+\frac{2 p(1-A)}{A}\right] g+\frac{p^{2}(1-A)^{2}}{A^{2}} f}{\frac{p^{2}}{A^{2}}},
\end{gathered}
$$

and

$$
u_{4}=\frac{t+\left[3+\frac{3 p(1-A)}{A}\right] h+\left[1+\frac{3 p(1-A)}{A}+\frac{3 p^{2}(1-A)^{2}}{A^{2}}\right] g+\frac{p^{3}(1-A)^{3}}{A^{3}} f}{\frac{p^{3}}{A^{3}}} .
$$


Now, we define the transformation $\Gamma: \mathbb{C}^{4} \times \mathbb{D} \longrightarrow \mathbb{C}$ as follows:

$$
\begin{aligned}
& \Gamma(f, g, h, t ; z)=¥\left(u_{1}, u_{2}, u_{3}, u_{4} ; z\right) \\
& =¥\left(f, \frac{g+\frac{p(1-A)}{A} f}{\frac{p}{A}}, \frac{h+\left[1+\frac{2 p(1-A)}{A}\right] g+\frac{p^{2}(1-A)^{2}}{A^{2}} f}{\frac{p^{2}}{A^{2}}},\right. \\
& \left.\frac{t+3\left[1+\frac{p(1-A)}{A}\right] h+\left[1+\frac{3 p(1-A)}{A}+\frac{3 p^{2}(1-A)^{2}}{A^{2}}\right] g+\frac{p^{3}(1-A)^{3}}{A^{3}} f}{\frac{p^{3}}{A^{3}}} ; z\right) .
\end{aligned}
$$

By utilizing Theorem 1 and Equations (33) to (36), and from Equation (40), we yield

$$
\Gamma\left(v(z), z v^{\prime}(z), z^{2} v^{\prime \prime}(z), z^{3} v^{\prime \prime \prime}(z) ; z\right)=¥\left(\mathcal{M}_{p}^{\ell} \vartheta(z), \mathcal{M}_{p}^{\ell+1} \vartheta(z), \mathcal{M}_{p}^{\ell+2} \vartheta(z), \mathcal{M}_{p}^{\ell+3} \vartheta(z) ; z\right) .
$$

Therefore, Equation (31) becomes

$$
\Gamma\left(v(z), z v^{\prime}(z), z^{2} v^{\prime \prime}(z), z^{3} v^{\prime \prime \prime}(z) ; z\right) \in \mathfrak{A} .
$$

A computation utilizing Equations (37), (38) and (39) acquire

$$
\frac{h}{q}+1=\frac{p\left[u_{3}-2(1-A) u_{2}+(1-A)^{2} u_{1}\right]}{A\left[u_{2}-(1-A) u_{1}\right]}
$$

and

$$
\frac{t}{q}=\frac{p^{2}\left[u_{4}-(1-A)^{3} u_{1}\right]-p[3 A+3 p(1-A)]\left[u_{3}-2(1-A) u_{2}+(1-A)^{2} u_{1}\right]}{A^{2}\left[u_{2}-(1-A) u_{1}\right]}-\frac{3 p^{2}(1-A)^{2}}{A^{2}}+2 .
$$

We also note that

$$
\left|\frac{z v^{\prime}(z)}{\omega^{\prime}(\chi)}\right|=\left|\frac{\frac{p}{A}\left[\mathcal{M}^{\ell+1}(z)-(1-A) \mathcal{M}^{\ell}(z)\right]}{\omega^{\prime}(\chi)}\right| \leq \kappa
$$

Hence, the admissibility condition for $¥ \in \Sigma_{\mathcal{M}}[\mathfrak{A}, \omega]$ in Definition 8 is equivalent to the admissibility condition of $\Gamma \in \Omega_{2}[\mathfrak{A}, \omega]$ as given in Definition 5 and by Theorem 1, we obtain

$$
\mathcal{M}_{p}^{\ell} \vartheta(z) \prec \omega(z)
$$

The proof of Theorem 2 is complete.

If $\mathfrak{A} \neq \mathbb{C}$ is a simply connected domain, then $\mathfrak{A}=\hbar(\mathbb{D})$ for some conformal mapping $\hbar(z)$ of $\mathbb{D}$ onto $\mathfrak{A}$. In this case the class $\Sigma_{\mathcal{M}}[\hbar(\mathbb{D}), \omega]$ is written as $\Sigma_{\mathcal{M}}^{\prime}[\hbar, \omega]$. The following outcome is a directly consequence of Theorem 2.

Theorem 3. Let $¥ \in \Sigma_{\mathcal{M}}[\hbar, \omega]$. If $\vartheta \in \mathcal{A}_{p}$ and $\omega \in \mathcal{J}_{0}$ achieve the following condition (28) given as follows:

$$
\Re\left(\frac{\chi \omega^{\prime \prime}(\chi)}{\omega^{\prime}(\chi)}\right) \geq 0, \quad\left|\frac{\mathcal{M}_{p}^{\ell+1} \vartheta(z)-(1-A) \mathcal{M}_{p}^{\ell} \vartheta(z)}{\omega^{\prime}(\chi)}\right| \leq \frac{|A| \kappa}{p}
$$


and

$$
¥\left(\mathcal{M}_{p}^{\ell} \vartheta(z), \mathcal{M}_{p}^{\ell+1} \vartheta(z), \mathcal{M}_{p}^{\ell+2} \vartheta(z), \mathcal{M}_{p}^{\ell+3} \vartheta(z) ; z\right) \prec h(z),
$$

then

$$
\mathcal{M}_{p}^{\ell} \vartheta(z) \prec \omega(z), \quad(z \in \mathbb{D}) .
$$

The next outcome is an extension of Theorem 3 to the case where the behavior of $\omega(z)$ on $\partial \mathbb{D}$ is not known.

Corollary 1. Let $\mathfrak{A} \subset \mathbb{C}$ and let $\omega(z)$ be univalent in $\mathbb{D}, \omega(0)=0$. Let $¥ \in \Sigma_{\mathcal{M}}\left[\mathfrak{A}, \omega_{\varepsilon}\right]$ for some $\varepsilon \in(0,1)$ where $\omega_{\varepsilon}(z)=\omega(\varepsilon z)$. If $\vartheta \in \mathcal{A}_{p}$ achieves

$$
\Re\left(\frac{\chi \omega_{\varepsilon}^{\prime \prime}(\chi)}{\omega_{\varepsilon}^{\prime}(\chi)}\right) \geq 0, \quad\left|\frac{\mathcal{M}_{p}^{\ell+1} \vartheta(z)-(1-A) \mathcal{M}_{p}^{\ell} \vartheta(z)}{\omega_{\varepsilon}^{\prime}(\chi)}\right| \leq \frac{|A| \kappa}{p},
$$

and

$$
¥\left(\mathcal{M}_{p}^{\ell} \vartheta(z), \mathcal{M}_{p}^{\ell+1} \vartheta(z), \mathcal{M}_{p}^{\ell+2} \vartheta(z), \mathcal{M}_{p}^{\ell+3} \vartheta(z) ; z\right) \in \mathfrak{A},
$$

then

$$
\mathcal{M}_{p}^{\ell} \vartheta(z) \prec \omega(z)
$$

where $z \in \mathbb{D}$ and $\chi \in \partial \mathbb{D} \backslash \mathcal{G}\left(\omega_{\varepsilon}\right)$.

Proof. By utilizing Theorem 3, we have $\mathcal{M}_{p}^{\ell} \vartheta(z) \prec \omega_{\varepsilon}(z)$. Then we get the outcome from $\omega_{\varepsilon}(z) \prec$ $\omega(z)$.

The next outcome is an immediate consequence of Corollary 1.

Corollary 2. Let $\mathfrak{A} \subset \mathbb{C}$ and let $\omega(z)$ be univalent in $\mathbb{D}, \omega(0)=0$. Let $¥ \in \Sigma_{\mathcal{M}}\left[\hbar, \omega_{\varepsilon}\right]$ for some $\varepsilon \in(0,1)$ where $\omega_{\varepsilon}(z)=\omega(\varepsilon z)$. If $\vartheta \in \mathcal{A}_{p}$ achieves

$$
\Re\left(\frac{\chi \omega_{\varepsilon}^{\prime \prime}(\chi)}{\omega_{\varepsilon}^{\prime}(\chi)}\right) \geq 0, \quad\left|\frac{\mathcal{M}_{p}^{\ell+1} \vartheta(z)-(1-A) \mathcal{M}_{p}^{\ell} \vartheta(z)}{\omega_{\varepsilon}^{\prime}(\chi)}\right| \leq \frac{|A| \kappa}{p},
$$

and

$$
¥\left(\mathcal{M}_{p}^{\ell} \vartheta(z), \mathcal{M}_{p}^{\ell+1} \vartheta(z), \mathcal{M}_{p}^{\ell+2} \vartheta(z), \mathcal{M}_{p}^{\ell+3} \vartheta(z) ; z\right) \prec \hbar(z),
$$

then

$$
\mathcal{M}^{\ell}(z) \prec \omega(z),
$$

where $z \in \mathbb{D}$ and $\chi \in \partial \mathbb{D} \backslash \mathcal{G}\left(\omega_{\varepsilon}\right)$.

The following outcome gives the best dominant of the differential subordination of Equation (40). 
Theorem 4. Let $\hbar(z)$ be univalent in $\mathbb{D}$. Let $¥: \mathbb{C}^{4} \times \mathbb{D} \longrightarrow \mathbb{C}$. Suppose that the differential equation:

$$
\begin{aligned}
& ¥\left(\omega(z), \frac{z \omega^{\prime}(z)+\frac{p(1-A)}{A} \omega(z)}{\frac{p}{A}}, \frac{z^{2} \omega^{\prime \prime}(z)+\left[1+\frac{2 p(1-A)}{A}\right] z \omega^{\prime}(z)+\frac{p^{2}(1-A)^{2}}{A^{2}} \omega(z)}{\frac{p^{2}}{A^{2}}},\right. \\
& \left.\frac{z^{3} \omega^{\prime \prime \prime}(z)+3\left[1+\frac{p(1-A)}{A}\right] z \omega^{\prime \prime}(z)+\left[1+\frac{3 p(1-A)}{A}+\frac{3 p^{2}(1-A)^{2}}{A^{2}}\right] z \omega^{\prime}(z)+\frac{p^{3}(1-A)^{3}}{A^{3}} \omega(z)}{\frac{p^{3}}{A^{3}}} ; z\right)=\hbar(z),
\end{aligned}
$$

has a solution $\omega(z)$ with $\omega(0)=0$ which achieves Equation (30). If $\vartheta \in \mathcal{A}_{p}$ achieves Equation (44) and

$$
¥\left(\mathcal{M}_{p}^{\ell} \vartheta(z), \mathcal{M}_{p}^{\ell+1} \vartheta(z), \mathcal{M}_{p}^{\ell+2} \vartheta(z), \mathcal{M}_{p}^{\ell+3} \vartheta(z) ; z\right),
$$

is holomorhic in $\mathbb{D}$, then

$$
\mathcal{M}_{p}^{\ell} \vartheta(z) \prec \omega(z)
$$

and $\omega(z)$ is the best dominant.

Proof. By utilizing Theorem 3 that $\omega(z)$ is a dominant of Equation (44). Since $\omega(z)$ achieves Equation (45), it is also a solution of Equation (44) and therefore $\omega(z)$ will be dominated by all dominants. Thus $\omega(z)$ is the best dominant.

In the case $\omega(z)=\mathcal{Q} z(\mathcal{Q}>0)$ and in view of Definition 8, the class of admissible functions $\Sigma_{\mathcal{M}}[\mathfrak{A}, \omega]$ denoted by $\Sigma_{\mathcal{M}}[\mathfrak{A}, \mathcal{Q}]$ is defined below:

Definition 7. Let $\mathfrak{A}$ be a set in $\mathbb{C}$ and $\mathcal{Q}>0$. The class of admissible functions $\Sigma_{\mathcal{M}}[\mathfrak{A}, \mathcal{Q}]$ consists of those functions $¥: \mathbb{C}^{4} \times \mathbb{D} \longrightarrow \mathbb{C}$ that achieve the admissibility condition

$$
\begin{aligned}
& ¥\left(\mathcal{Q} e^{i \theta},\left[\frac{\kappa A}{p}+(1-A)\right] \mathcal{Q} e^{i \theta}, \frac{\mathcal{L}+\left[\left[1+\frac{2 p(1-A)}{A}\right] \kappa+\frac{p^{2}(1-A)^{2}}{A^{2}}\right] \mathcal{Q} e^{i \theta}}{\frac{p^{2}}{A^{2}}},\right. \\
& \left.\frac{\mathcal{V}+\left[1+\frac{p(1-A)}{A}\right] 3 \mathcal{L}+\left[\left[1+\frac{3 p(1-A)}{A}+\frac{3 p^{2}(1-A)^{2}}{A^{2}}\right] \kappa+\frac{p^{3}(1-A)^{3}}{A^{3}}\right] \mathcal{Q} e^{i \theta}}{\frac{p^{3}}{A^{3}}}\right) \notin \mathfrak{A},
\end{aligned}
$$

where $z \in \mathbb{D}, \Re\left(\mathcal{L} e^{-i \theta}\right) \geq(\kappa-1) \kappa \mathcal{Q}$ and $\Re\left(\mathcal{V} e^{-i \theta}\right) \geq 0$ for all real $\theta$ and $\kappa \in \mathbb{N} \backslash\{1\}$.

Corollary 3. Let $¥ \in \Sigma_{\mathcal{M}}[\mathfrak{A}, \mathcal{Q}]$. If $\vartheta \in \mathcal{A}_{p}$ achieves the following conditions:

$$
\left|\mathcal{M}_{p}^{\ell+1} \vartheta(z)-(1-A) \mathcal{M}_{p}^{\ell} \vartheta(z)\right| \leq \frac{|A| \kappa \mathcal{Q}}{p}
$$

and

$$
¥\left(\mathcal{M}_{p}^{\ell} \vartheta(z), \mathcal{M}_{p}^{\ell+1} \vartheta(z), \mathcal{M}_{p}^{\ell+2} \vartheta(z), \mathcal{M}_{p}^{\ell+3} \vartheta(z) ; z\right) \in \mathfrak{A},
$$

then

$$
\left|\mathcal{M}^{\ell}(z)\right|<\mathcal{Q}(z) .
$$


In the case $\mathfrak{A}=\omega(\mathbb{D})=\left\{z_{1}:\left|z_{1}\right|<\mathcal{Q}\right\},(\mathcal{Q}>0)$, for simplification we denote by $\Sigma_{\mathcal{M}}[\mathcal{Q}]$ to the class $\Sigma_{\mathcal{M}}[\mathfrak{A}, \mathcal{Q}]$.

Corollary 4. Let $¥ \in \Sigma_{\mathcal{M}}[\mathcal{Q}]$. If $\vartheta \in \mathcal{A}_{p}$ achieves the following conditions

$$
\left|\mathcal{M}_{p}^{\ell+1} \vartheta(z)-(1-A) \mathcal{M}_{p}^{\ell} \vartheta(z)\right| \leq \frac{|A| \kappa \mathcal{Q}}{p},
$$

and

$$
\left|¥\left(\mathcal{M}_{p}^{\ell} \vartheta(z), \mathcal{M}^{\ell+1}(z), \mathcal{M}_{p}^{\ell+2} \vartheta(z), \mathcal{M}_{p}^{\ell+3} \vartheta(z) ; z\right)\right|<\mathcal{Q},
$$

then

$$
\left|\mathcal{M}_{p}^{\ell} \vartheta(z)\right|<\mathcal{Q} .
$$

Corollary 5. If $\kappa \geq 2, \mathcal{Q}>0$. If $\vartheta \in \mathcal{A}_{p}$ achieves

$$
\left|\mathcal{M}_{p}^{\ell+1} \vartheta(z)-\mathcal{M}_{p}^{\ell} \vartheta(z)\right|<\frac{(\kappa-p)|A| \mathcal{Q}}{p},
$$

then

$$
\left|\mathcal{M}_{p}^{\ell} \vartheta(z)\right|<\mathcal{Q}
$$

Proof. Let $¥\left(u_{1}, u_{2}, u_{3}, u_{4} ; z\right)=u_{2}-u_{1}$. Utilizing Corollary 3 with $\mathfrak{A}=\hbar(\mathbb{D})$ and

$$
\hbar(z)=\frac{(\kappa-p)|A| \mathcal{Q}}{p} z, \quad(\mathcal{Q}>0, z \in \mathbb{D}) .
$$

We have to find the condition so that $¥ \in \Sigma_{\mathcal{M}}[\mathfrak{A}, \mathcal{Q}]$, that is, the admissibility condition of Equation (48) is achieved. This follows since

$$
\begin{aligned}
& \mid ¥\left(\mathcal{Q} e^{i \theta},\left[\frac{\kappa A}{p}+(1-A)\right] \mathcal{Q} e^{i \theta}, \frac{\mathcal{L}+\left[\left[1+\frac{2 p(1-A)}{A}\right] \kappa+\frac{p^{2}(1-A)^{2}}{A^{2}}\right] \mathcal{Q} e^{i \theta}}{\frac{p^{2}}{A^{2}}},\right. \\
& \left.\frac{\mathcal{V}+\left[1+\frac{p(1-A)}{A}\right] 3 \mathcal{L}+\left[\left[1+\frac{3 p(1-A)}{A}+\frac{3 p^{2}(1-A)^{2}}{A^{2}}\right] \kappa+\frac{p^{3}(1-A)^{3}}{A^{3}}\right] \mathcal{Q} e^{i \theta}}{\frac{p^{3}}{A^{3}}}\right) \mid \\
& =\left|\left[\frac{\kappa}{p}-1\right] A \mathcal{Q} e^{i \theta}\right|=\frac{(\kappa-p)|A| \mathcal{Q}}{p} .
\end{aligned}
$$

The required outcome is obtained.

Corollary 6. If $\kappa \geq 2, \mathcal{Q}>0$. If $\vartheta \in \mathcal{A}_{p}$ achieves

$$
\left|\mathcal{M}_{p}^{\ell+2} \vartheta(z)-\mathcal{M}_{p}^{\ell+1} \vartheta(z)\right|<\frac{\left[2\left(1+\left|1+\frac{p(1-2 A)}{A}\right|\right)+\left|\frac{p^{2}(A-1)}{A}\right|\right] \mathcal{Q}}{\frac{p^{2}}{|A|^{2}}}
$$


then

$$
\left|\mathcal{M}_{p}^{\ell} \vartheta(z)\right|<\mathcal{Q}
$$

Proof. Let $¥\left(u_{1}, u_{2}, u_{3}, u_{4} ; z\right)=u_{3}-u_{2}$. Utilizing Corollary 3 with $\mathfrak{A}=\hbar(\mathbb{D})$ and

$$
\hbar(z)=\frac{\left[2\left(1+\left|1+\frac{p(1-2 A)}{A}\right|\right)+\left|\frac{p^{2}(A-1)}{A}\right|\right] \mathcal{Q}}{\frac{p^{2}}{|A|^{2}}} z, \quad(\mathcal{Q}>0, z \in \mathbb{D}) .
$$

It is enough to show that $¥ \in \Sigma_{\mathcal{M}}[\mathfrak{A}, \mathcal{Q}]$, that is, the admissibility condition of Equation (48) is achieved. This follows since

$$
\begin{aligned}
& \mid ¥\left(\mathcal{Q} e^{i \theta},\left[\frac{\kappa A}{p}+(1-A)\right] \mathcal{Q} e^{i \theta}, \frac{\mathcal{L}+\left[\left[1+\frac{2 p(1-A)}{A}\right] \kappa+\frac{p^{2}(1-A)^{2}}{A^{2}}\right] \mathcal{Q} e^{i \theta}}{\frac{p^{2}}{A^{2}}},\right. \\
& \left.\frac{\mathcal{V}+\left[1+\frac{p(1-A)}{A}\right] 3 \mathcal{L}+\left[\left[1+\frac{3 p(1-A)}{A}+\frac{3 p^{2}(1-A)^{2}}{A^{2}}\right] \kappa+\frac{p^{3}(1-A)^{3}}{A^{3}}\right] \mathcal{Q} e^{i \theta}}{\frac{p^{3}}{A^{3}}}\right) \mid \\
& =\left|\frac{\mathcal{L}+\left[1+\frac{p(1-2 A)}{A}\right] \kappa \mathcal{Q} e^{i \theta}+\left[\frac{p^{2}(A-1)}{A}\right] \mathcal{Q} e^{i \theta}}{\frac{p^{2}}{A^{2}}}\right|=\left|\frac{\mathcal{L} e^{-i \theta}+\left[1+\frac{p(1-2 A)}{A}\right] \kappa \mathcal{Q}+\left[\frac{p^{2}(A-1)}{A}\right] \mathcal{Q} \mid}{\frac{p^{2}}{A^{2}} e^{-i \theta}}\right| \\
& \geq \frac{\Re\left(\mathcal{L} e^{-i \theta}\right)+\left|1+\frac{p(1-2 A)}{A}\right| \kappa \mathcal{Q}+\left|\frac{p^{2}(A-1)}{A}\right| \mathcal{Q}}{\frac{p^{2}}{|A|^{2}}} \geq \frac{\left[2\left(1+\left|1+\frac{p(1-2 A)}{A}\right|\right)+\left|\frac{p^{2}(A-1)}{A}\right|\right] \mathcal{Q}}{\frac{p^{2}}{|A|^{2}}} .
\end{aligned}
$$

This completes the proof.

Corollary 7. If $\kappa \geq 2, \mathcal{Q}>0$. If $\vartheta \in \mathcal{A}_{p}$ achieves

$\left|\mathcal{M}_{p}^{\ell+2} \vartheta(z)-\mathcal{M}_{p}^{\ell+1} \vartheta(z)\right|<\frac{\left[2\left(\left|2+\frac{3 p(1-A)}{A}\right|+\left|\frac{p(1-A)}{A}\left(1+\frac{3 p(1-A)}{A}\right)\right|\right)+\left|\frac{p^{2}(1-A)^{2}}{A^{2}}\left(\frac{p(1-A)}{A}-1\right)\right|\right] \mathcal{Q}}{\frac{p^{3}}{|A|^{3}}}$,

then

$$
\left|\mathcal{M}_{p}^{\ell} \vartheta(z)\right|<\mathcal{Q}
$$

Proof. Let $¥\left(u_{1}, u_{2}, u_{3}, u_{4} ; z\right)=u_{4}-\frac{A}{p} u_{3}$. Using Corollary 3 with $\mathfrak{A}=\hbar(\mathbb{D})$ and

$$
\hbar(z)=\frac{\left[2\left(\left|2+\frac{3 p(1-A)}{A}\right|+\left|\frac{p(1-A)}{A}\left(1+\frac{3 p(1-A)}{A}\right)\right|\right)+\left|\frac{p^{2}(1-A)^{2}}{A^{2}}\left(\frac{p(1-A)}{A}-1\right)\right|\right] \mathcal{Q}}{\frac{p^{3}}{|A|^{3}}} z, \quad(\mathcal{Q}>0, z \in \mathbb{D}) .
$$


It is adequate to show that $¥ \in \Sigma_{\mathcal{M}}[\mathfrak{A}, \mathcal{Q}]$, that is, the admissibility condition of Equation (48) is achieved. This follows since

$$
\begin{aligned}
& ¥\left(\mathcal{Q} e^{i \theta},\left[\frac{\kappa A}{p}+(1-A)\right] \mathcal{Q} e^{i \theta}, \frac{\mathcal{L}+\left[\left[1+\frac{2 p(1-A)}{A}\right] \kappa+\frac{p^{2}(1-A)^{2}}{A^{2}}\right] \mathcal{Q} e^{i \theta}}{\frac{p^{2}}{A^{2}}},\right. \\
& \left.\frac{\mathcal{V}+\left[1+\frac{p(1-A)}{A}\right] 3 \mathcal{L}+\left[\left[1+\frac{3 p(1-A)}{A}+\frac{3 p^{2}(1-A)^{2}}{A^{2}}\right] \kappa+\frac{p^{3}(1-A)^{3}}{A^{3}}\right] \mathcal{Q} e^{i \theta}}{\frac{p^{3}}{A^{3}}}\right) \mid \\
& =\left|\frac{\mathcal{V}+\left[2+\frac{3 p(1-A)}{A}\right] \mathcal{L}+\left[\frac{p(1-A)}{A}\left(1+\frac{3 p(1-A)}{A}\right)\right] \kappa \mathcal{Q} e^{i \theta}+\left[\frac{p^{2}(1-A)^{2}}{A^{2}}\left(\frac{p(1-A)}{A}-1\right)\right] \mathcal{Q} e^{i \theta}}{\frac{p^{3}}{A^{3}}}\right| \\
& =\left|\frac{\mathcal{V} e^{-i \theta}+\left[2+\frac{3 p(1-A)}{A}\right] \mathcal{L} e^{-i \theta}+\left[\frac{p(1-A)}{A}\left(1+\frac{3 p(1-A)}{A}\right)\right] \kappa \mathcal{Q}+\left[\frac{p^{2}(1-A)^{2}}{A^{2}}\left(\frac{p(1-A)}{A}-1\right)\right] \mathcal{Q}}{\frac{p^{3}}{A^{3}} e^{-i \theta}}\right| \\
& \geq \frac{\Re\left(\mathcal{V} e^{-i \theta}\right)+\left|2+\frac{3 p(1-A)}{A}\right| \Re\left(\mathcal{L} e^{-i \theta}\right)+\left|\frac{p(1-A)}{A}\left(1+\frac{3 p(1-A)}{A}\right)\right| \kappa \mathcal{Q}+\left|\frac{p^{2}(1-A)^{2}}{A^{2}}\left(\frac{p(1-A)}{A}-1\right)\right| \mathcal{Q}}{\frac{p^{3}}{|A|^{3}}} \\
& \geq \frac{(\kappa-1) \kappa \mathcal{Q}\left|2+\frac{3 p(1-A)}{A}\right|+\left|\frac{p(1-A)}{A}\left(1+\frac{3 p(1-A)}{A}\right)\right| \kappa \mathcal{Q}+\left|\frac{p^{2}(1-A)^{2}}{A^{2}}\left(\frac{p(1-A)}{A}-1\right)\right| \mathcal{Q}}{\frac{p^{3}}{|A|^{3}}} \\
& \geq \frac{\left[2\left(\left|2+\frac{3 p(1-A)}{A}\right|+\left|\frac{p(1-A)}{A}\left(1+\frac{3 p(1-A)}{A}\right)\right|\right)+\left|\frac{p^{2}(1-A)^{2}}{A^{2}}\left(\frac{p(1-A)}{A}-1\right)\right|\right] \mathcal{Q}}{\frac{p^{3}}{|A|^{3}}}
\end{aligned}
$$

The required outcome is derived.

\section{Conclusions and Future Directions}

In the terms of the $\xi$-Generalized Hurwitz-Lerch Zeta functions (GHLZF) in the z- domain, a new generalized Noor-type linear integral operator is introduced. This operator was utilized to study new classes of holomorphic functions in $\mathbb{D}$. In addition, new applications of the third-order differential subordination outcome that involves this new operator were investigated. The third-order differential inequalities were imposed in this work to show the uppercase of this new generalized Noor-type linear integral operator in $\mathbb{D}$.

Author Contributions: H.A.-J. writing the original draft; F.G. and M.D. writing review and editing. All authors have read and agreed to the published version of the manuscript.

Funding: This research was funded by Universiti Kebangsaan Malaysia, grant number GUP-2019-032.

Conflicts of Interest: The authors declare no conflict of interest. 


\section{References}

1. Duren, P.L. Univalent Functions; Springer: New York, NY, USA, 1983.

2. Koebe, P. Über die Uniformisierung beliebiger analytischer kurven. Nachrichten Ges. Wiss. Göttingen. Math. Phys. Kl. 1907, 1907, 191-210.

3. Koebe, P. Über eine neue Methode der konformen Abbildung und Uniformisierung. Nachrichten von der Gesellschaft der Wissenschaften zu Göttingen Mathematisch-Physikalische Klasse 1912, 1912, 844-848.

4. Goodman, A.W. Univalent Functions, I; Mariner: Tampa, FL, USA, 1983.

5. Littlewood, J.E. On equalities in the theory of functions. Proc. Lond. Math. Soc. 1925, 23, 481-519. [CrossRef]

6. Littlewood, J.E. Lectures on the Theory of Functions; Oxford University Press: Oxford, UK; London, UK, 1944.

7. Rogosinski, W. On subordinate functions. Proc. Camb. Philos. Soc. 1939, 35, 1-26. [CrossRef]

8. Rogosinski, W. On the coefficients of subordinate functions. Proc. London Math. Soc. 1945, 48, 48-82. [CrossRef]

9. Goluzin, G.M. On the majorization principle in function theory (Russian). Dokl. Akad. Nauk. SSSR 1953, 42, 647-650.

10. Suffridge, T.J. Some remarks on convex maps of the unit disk. Duke Math. J. 1970, 37, 775-777. [CrossRef]

11. Robinson, R.M. Univalent majorants. Trans. Am. Math. Soc. 1947, 61, 1-35. [CrossRef]

12. Miller, S.S.; Mocanu, P.T. Differential subordinations and univalent functions. Michigan Math. J. 1981, 28, 157-171. [CrossRef]

13. Miller, S.S.; Mocanu, P.T. On some classes of first order differential subordinations. Michigan Math. J. 1985, 32, 185-195. [CrossRef]

14. Miller, S.S.; Mocanu, P.T. Differential subordinations and inequalities in the complex plane. J. Differ. Eqns. 1987, 67, 199-211. [CrossRef]

15. Miller, S.S.; Mocanu, P.T. The theory and applications of second-order differential subordinations. Studia Univ. Babeş-Bolyai Math. 1989, 34, 3-33.

16. Miller, S.S.; Mocanu, P.T. A Special Differential Subordination and Its Application to Univalency Conditions. In Current Topics in Analytic Function Theory; World Scientific: Singapore; London, UK, 1992; pp. 171-185.

17. Miller, S.S.; Mocanu, P.T. Briot-Bouquet differential equations and differential subordinations. Complex Var. 1997, 33, 217-237. [CrossRef]

18. Ponnusamy, S.; Juneja, O.P. Third-Order Differential Inequalities in the Complex Plane. In Current Topics in Analytic Function Theory; World Scientific: Singapore; London, UK, 1992.

19. Miller, S.S.; Mocanu, P.T. Differential Subordinations: Theory and Applications; Marcel Dekker: New York, NY, USA, 2000.

20. Antonino, J.A.; Miller, S.S. Third-order differential inequalities and subordinations in the complex plane. Complex Var. Appl. 2011, 56, 439-454. [CrossRef]

21. Tang, H.; Srivastiva, H.M.; Li, S.; Ma, L. Third-order differential subordinations and superordination results for meromorphically multivalent functions associated with the Liu-Srivastava Operator. Abstr. Appl. Anal. 2014, 2014, 1-11. [CrossRef]

22. Tang, H.; Deniz, E. Third-order differential subordinations results for analytic functions involving the generalized Bessel functions. Acta Math. Sci. 2014, 6, 1707-1719. [CrossRef]

23. Farzana, H.A.; Stephen, B.A.; Jeyaraman, M.P. Certain third-order differential subordination and superordination results of meromorphic multivalent functions. Asia Pacific J. Math. 2015, 2, $76-87$.

24. Tang, H.; Srivastiva, H.M.; Deniz, E.; Li, S.-H. Third-order differential superordination involving the generalized Bessel functions. Bull. Malay. Math. Soc. 2015, 38, 1669-1688. [CrossRef]

25. Ibrahim, R.W.; Ahmad, M.Z.; Al-Janaby, H.F. The Third-Order Differential Subordination and Superordination involving a fractional operator. Open Math. 2015, 13, 706-728. [CrossRef]

26. El-Ashwah, R.M.; Hassan, A.H. Some third-order differential subordination and superordination results of some meromorphic functions using a Hurwitz-Lerech Zeta type operator. Ilirias J. Math. 2015, 4, 1-15.

27. El-Ashwah, R.M.; Hassan, A.A. Third-order differential subordination and superordination results by using Fox-Wright generalized hypergeometric function. Func. Anal. TMA 2016, 2, 34-51.

28. Attiya, A.A.; Kwon, O.S.; Hyang, P.J.; Cho, N.E. An integrodifferential operator for meromorphic functions associated with the Hurwitz-Lerch Zeta function. Filomat 2016, 30, 2045-2057. [CrossRef] 
29. Răducanu, D. Third-order differential subordinations for analytic functions associated with generalized Mittag-Leffler functions. Mediterr. J. Math. 2017, 14, 1-18. [CrossRef]

30. Srivastava, H.M.; Prajapati, A.; Gochhayat, P. Third-order differential subordination and differential superordination results for analytic functions involving the Srivastava-Attiya operator. Appl. Math. Inf. Sci. 2018, 12, 469-481. [CrossRef]

31. Gochhayat, P.; Prajapati, A. Applications of third order differential subordination and superordination involving generalized Struve function. Filomat 2019, 33, 3047-3059. [CrossRef]

32. Alexander, J.W. Functions which map the interior of the unit circle upon simple regions. Ann. Math. 1915, 17, 12-22. [CrossRef]

33. Libera, R.J. Some classes of regular univalent functions. Proc. Am. Math. Soc. 1965, 16, 755-758. [CrossRef]

34. Bernardi, S.D. Convex and starlike univalent functions. Trans. Am. Math. Soc. 1969, 135, 429-446. [CrossRef]

35. Ruscheweyh, S. New criteria for univalent functions. Proc. Am. Math. Soc. 1975, 49, 109-115. [CrossRef]

36. Noor, K.L. On new classes of integral operators. J. Nat. Geom. 1999, 16, 71-80.

37. Cho, N.E.; Saigo, M.; Srivastava, H.M. Some inclusion properties of a certain family of integral operators. J. Math. Anal. Appl. 2002, 276, 432-445. [CrossRef]

38. Liu, J.-L.; Noor, K.I. Some properties of noor integral operator. J. Nat. Geom. 2002, 21, 81-90.

39. Branges, L.D. A proof of the Bieberbach conjecture. Acta Math. 1984, 154, 137-152. [CrossRef]

40. Al-Janay, H.F.; Ghanim, F. On Subclass Noor-Type Harmonic Multivalent Functions Based on Hypergeometric Functions. Kragujev. J. Math. 2020, 45, 499-519.

41. Al-Janaby, H.F.; Ghanim, F.; Ahmad, M.Z. Harmonic Multivalent Functions Associated with an Extended Generalized Linear Operator of Noor-type. J. Nonlinear Funct. Anal. Appl. 2019, 24, 269-292.

42. Al-Janaby, H.F.; Ghanim, F.; Agarwal, P. Geometric Studies on Inequalities of Harmonic Functions in a Complex Field Based on $\xi$-Generalized Hurwitz-Lerch Zeta Function Iran. J. Math. Sci. Inform. 2020, accepted.

43. Al-Janay, H.F.; Ghanim, F.; Darus, M. Some Geometric Properties of Integral Operators Proposed by Hurwitz-Lerch Zeta Function. IOP Conf. Ser. J. Phys. Conf. Ser. 2019, 1212, 1-6.

44. Ghanim, F. Study of a certain subclass of Hurwitz-Lerch zeta function related to a linear operator. Abstr. Appl. Anal. 2013, 2013, 1-7. [CrossRef]

45. Ghanim, F.; Darus, M. New result of analytic functions related to Hurwitz-zeta function. Sci. World J. 2013, 2013, 1-5. [CrossRef]

46. Ghanim, F.; Al-Janaby, H.F. A Certain Subclass of Univalent Meromorphic Functions Defined by a Linear Operator Associated with the Hurwitz-Lerch Zeta Function. Rad Hrvat. Akad. Znan. Umjet. Mat. Znan. 2019, 23, 71-83. [CrossRef]

47. Răducanu, D.; Srivastava, H.M. A new class of analytic functions defined by means of a convolution operator involving the Hurwitz-Lerch zeta function. Integr. Trans. Spec. Funct. 2007, 18, 933-943. [CrossRef]

48. Srivastava, H.M.; Attiya, A.A. An integral operator associated with the Hurwitz-Lerch zeta function and differential subordination. Integr. Trans. Spec. Funct. 2007, 18, 207-216. [CrossRef]

49. Srivastava, H.M.; Gaboury, S.A.; Ghanim, F. Certain subclasses of meromorphically univalent functions defined by a linear operator associated with the $\lambda$-generalized Hurwitz-Lerch zeta function. Integr. Transf. Spec. Funct. 2015, 26, 258-272. [CrossRef]

50. Srivastava, H.M.; Gaboury, S.A.; Ghanim, F. Some further properties of a linear operator associated with the $\lambda$-generalized Hurwitz-Lerch zeta function related to the class of meromorphically univalent functions. Appl. Math. Comput. 2015, 259, 1019-1029.

51. Xing, S.C.; Jose, L.L. A note on the asymptotic expansion of the Lerch's transcendent. Integr. Trans. Spec. Funct. 2019, 30, 844-855.

52. Choi, J.; Srivastava, H.M. The Multiple Hurwitz Zeta Function and the Multiple Hurwitz-Euler Eta Function. Taiwan J. Math. 2011, 15, 501-522. [CrossRef]

53. Milovanovic, G.V.; Rassias, M.T. (Eds.) Analytic Number Theory, Approximation Theory and Special Functions-In Honor of Hari M. Srivastava; Springer: Basel, Switzerland, 2014.

54. Rassias, M.T.; Yang, B. On an Equivalent Property of a Reverse Hilbert-Type Integral Inequality Related to the Extended Hurwitz-Zeta Function. J. Math. Inequal. 2019, 13, 315-334. [CrossRef]

55. Rassias, M.T.; Yang, B. On a Hilbert-type integral inequality related to the extended Hurwitz zeta function in the whole plane. Acta Appl. Math. 2019, 160, 67-80. [CrossRef] 
56. Rassias, M.T.; Yang, B. Equivalent properties of a Hilbert-type integral inequality with the best constant factor related to the Hurwitz zeta function. Ann. Funct. Anal. 2018, 9, 282-295. [CrossRef]

57. Rassias, M.T.; Yang, B.; Raigorodskii, A. Two Kinds of the Reverse Hardy-Type Integral Inequalities with the Equivalent Forms Related to the Extended Riemann Zeta Function. Appl. Anal. Discret. Math. 2018, 12, 273-296. [CrossRef]

58. Srivastava, H.M.; Choi, J. Series Associated with Zeta and Related Functions; Kluwer Academic Publishers: Dordrecht, Germany, 2001.

59. Srivastava, H.M. Some formulas for the Bernoulli and Euler polynomials at rational arguments. Math. Proc. Camb. Philos. Soc. 2000, 129, 77-84. [CrossRef]

60. Srivastava, H.M.; Choi, J. Zeta and q-zeta Functions and Associated Series and Integrals; Elsevier Science Publishers: Amsterdam, The Netherland, 2012.

61. Srivastava, H.M. A new family of the $\lambda$-generalized Hurwitz-Lerch zeta functions with applications. Appl. Math. Inf. Sci. 2014, 8, 1485-1500. [CrossRef]

62. Mathai, A.M.; Saxena, R.K.; Haubold, H.J. The H-function: Theory and applications; Springer: New York, NY, USA, 2010.

63. Srivastava, H.M.; Gupta, K.C.; Goyal, S.P. The H-functions of One and Two Variables with Applications; South Asian Publishers: New Delhi, India, 1982.

64. Srivastava, H.M.; Gaboury, S.; Tremblay, R. New relations involving an extended multiparameter Hurwitz-Lerch zeta function with applications. Int. J. Anal. 2014, 2014, 1-14. [CrossRef]

65. Cho, N.E.; Kwon, O.S.; Srivastava, H.M. Inclusion relationships and argument properties for certain subclasses of multivalent functions associated with a family of linear operators. J. Math. Anal. Appl. 2004, 292, 470-483. [CrossRef]

66. Noor, K.L. Integral operators defined by convolution with hypergeometric functions. Appl. Math. Comput. 2006, 182, 1872-1881. [CrossRef]

67. Darus, M.; Ibrahim, R.W. Integral operator defined by convolution product of hypergeometric functions. Int. J. Nonlinear Sci. 2012, 13, 153-157.

(C) 2020 by the authors. Licensee MDPI, Basel, Switzerland. This article is an open access article distributed under the terms and conditions of the Creative Commons Attribution (CC BY) license (http://creativecommons.org/licenses/by/4.0/). 